\title{
Diacronie
}

Studi di Storia Contemporanea

$\mathrm{N}^{\circ} 15,3 \mid 2013$

Spazi, percorsi e memorie

\section{Filippo Focardi, Il cattivo tedesco e il bravo italiano. La rimozione delle colpe della seconda guerra mondiale}

\section{Oreste Veronesi}

\section{(2) OpenEdition}

Journals

Edizione digitale

URL: http://journals.openedition.org/diacronie/631

DOI: 10.4000/diacronie.631

ISSN: 2038-0925

Editore

Association culturelle Diacronie

Notizia bibliografica digitale

Oreste Veronesi, « Filippo Focardi, Il cattivo tedesco e il bravo italiano. La rimozione delle colpe della seconda guerra mondiale », Diacronie [Online], $N^{\circ} 15,3$ | 2013, documento 17, Messo online il 01 octobre 2013, consultato il 22 septembre 2020. URL : http://journals.openedition.org/diacronie/631 ; DOI : https://doi.org/10.4000/diacronie.631 


\section{Diacronie}

\section{RECENSIONE:}

\section{Filippo FOCARDI, Il cattivo tedesco e il bravo italiano. La rimozione delle colpe della seconda guerra mondiale, Roma - Bari, Laterza, 2013, XIX + 288 pp.}

a cura di Oreste VERONESI *

Nel corso degli anni si è consolidata nell'uso pubblico della storia un'immagine del soldato italiano reticente di fronte ad una realtà dei fatti che, grazie a diversi storici - e in particolare a partire dagli anni Settanta - ha messo in mostra la gravità delle occupazioni fasciste. Anche gli studi sul fascismo sembrano non permeare il dibattito pubblico, più incline all'utilizzazione di cliché che ne sminuiscono la portata degli eventi. Basti pensare in questo senso alle ultime tristi affermazioni di Roberta Lombardi o Silvio Berlusconi, che si riferiscono al fascismo come fenomeno sano fino all'alleanza con Hitler, poi degenerato e quindi condannabile. Un problema messo in evidenza di recente da Eric Gobetti, che ha parlato di «rifiuto collettivo della memoria del ventennio e della guerra fascista ${ }^{1}$.

Per capire come questa serie di costruzioni stereotipate abbia potuto radicarsi così saldamente nell'immaginario collettivo ci viene in aiuto Filippo Focardi, con il suo ultimo lavoro frutto di una ricerca ventennale. Il libro, infatti, è una rielaborazione della tesi di dottorato dell'autore, nonché di numerosi articoli apparsi negli anni in diverse riviste $^{2}$. Focardi, ricercatore di Storia contemporanea presso il Dipartimento di Scienze politiche, giuridiche e studi internazionali dell'Università di Padova, alle sue spalle ha già alcuni libri pubblicati che si inseriscono all'interno del suo percorso di

\footnotetext{
${ }^{1}$ GOBETTI Eric, «L'occupazione italiana in Jugoslavia (1941-1943). Storiografia e memoria pubblica», in Passato Presente, 87, 3/2012, pp. 39-53.

${ }^{2}$ Si vedano ad esempio: FOCARDI, Filippo, «"Bravo italiano" e "cattivo tedesco": riflessioni sulla genesi di due immagini incrociate», in Storia e Memoria, 1, 1/1996, pp. 55-83; ID., "La memoria della guerra e il mito del "bravo italiano": origine e affermazione di un autoritratto collettivo», in Italia Contemporanea, 220-221, 3/2000, pp. 393-399.
} 
ricerca. Per Laterza (2005) è stato autore del volume La guerra della memoria. La resistenza nel dibattito politico italiano dal 1945 a oggi3 in cui è già possibile trovare accenni alla ricerca che qui recensiamo, mentre per Carocci (2008) ha pubblicato Criminali di guerra in libertà 4 .

La ricerca di Focardi si inserisce in un più ampio contesto storiografico sulla storia del colonialismo fascista. Se negli anni Settanta sono stati in particolare i lavori di Enzo Collotti, Angelo Del Boca e Giorgio Rochat a dare il via alla ricerca, negli ultimi anni le pubblicazioni in merito sono fiorite. Recentemente è stato pubblicato l'ultimo lavoro di Eric Gobetti per Laterza Attenti al nemico. L'occupazione italiana in Jugoslavia (19411943). Un lavoro più organico è invece quello di Elena Aga-Rossi e Maria Teresa Giusti Una guerra a parte. I militari italiani nei Balcani 1940-19455. Passando per Angelo Del Boca, Costantino Di Sante, Alessandra Kersevan, Davide Conti etc., i lavori sono davvero numerosi. Tuttavia, come abbiamo già detto, a dispetto di questa prolifica bibliografia, persistono diversi stereotipi. Nonostante alcuni ottimi risultati editoriali come ad esempio le ricerche di Gianni Oliva, quale «Si ammazza troppo poco». I crimini di guerra italiani $1940-1943^{6}$ o, in campo non storiografico, i libri di Boris Pahor7, jugoslavo italianizzato durante il fascismo, stenta a diffondersi una cultura consapevole dei crimini di guerra italiani.

È in questo contesto che si inserisce il lavoro di Filippo Focardi e qui ne spicca l'originalità. L'autore, tuttavia, non intende inserirsi in questo ampio repertorio andando ad aggiungere qualche tassello alla disamina dei crimini o dei criminali di guerra italiani. L'obiettivo è quello di declinare nel contesto italiano, tra storia politica e storia culturale, le ricerche dello storico Tony $\mathrm{Judt}^{8}$ in merito alla costruzione europea della memoria del conflitto. Come scrive l'autore nell'introduzione «questo libro

3 FOCARDI, Filippo, La guerra della memoria. La resistenza nel dibattito politico italiano dal 1945 a oggi, Roma-Bari, Laterza, 2005.

4 ID., Criminali di guerra in libertà. Un accordo segreto tra Italia e Germania federale, 19491955, Roma, Carocci, 2008.

5 GOBETTI, Eric, Alleati del nemico. L'occupazione italiana in Jugoslavia (1941-1943), RomaBari, Laterza, 2013; AGA-ROSSI, Elena, GIUSTI, Maria Teresa, Una guerra a parte. I militari italiani nei balcani 1940-1945, Bologna, Il Mulino, 2011.

6 OLIVA, Gianni, «Si ammazza troppo poco». I crimini di guerra italiani 1940-1943, Milano, Mondadori, 2007.

7 PAHOR, Boris, ORLIC, Mila, Tre volte no. Memorie di un uomo libero, Milano, Rizzoli, 2009; PAHOR, Boris, Figlio di nessuno, un'autobiografia senza frontiere, Milano, Rizzoli, 2012.

${ }^{8}$ Lo storico britannico afferma infatti, in un articolo del 2000 a cui Focardi fa direttamente riferimento, quanto segue: «I shall suggest that the ways in which the official versions of the war and postwar era have unraveled in recent years are indicative of unresolved problems that lie at the center of the present continental crisis». Cfr. JUDT, Tony, Tha past is another Country: Myth and Memory in Postwar Europe, in DEAK, Istvàn, GROSS, Jan T., JUDT, Tony (eds.), The politics of Retribution in Europe. World War II and its Aftermath, Princeton, Princeton University Press, 2000, pp. 293-294. 
intende ricostruire lo specifico percorso di costruzione di una narrazione italiana dell'esperienza della seconda guerra mondiale»9. L'opera è dunque una puntuale disamina di quel fenomeno revisionista che «è già avvertibile nel nostro paese nei primi anni del dopoguerra quando cominciano ad apparire le memorie o i diari dei capi militari che hanno condotto e perso la guerra e dei massimi gerarchi fascisti $»^{10}$ e che si è consolidato anche grazie alla reticenza dei manuali scolastici ${ }^{11}$.

Il volume è diviso in sette capitoli che intendono affrontare diversi nodi tematici in riferimento alla costruzione della memoria italiana. Il primo capitolo ci introduce brevemente nella narrazione della propaganda alleata, una pomposa macchina mediatica che trovava nella radio il suo mezzo più efficace. L'intento alleato fu innanzitutto quello di dividere regime e popolo italiano, per scaricare sul primo tutte le responsabilità e cercare quindi di minarne il consenso tentando di far identificare il Duce e il fascismo come traditori del popolo italiano. In particolare attraverso Radio Londra la narrazione degli alleati cercò di costruire l'essenza di un'italianità vicina al popolo britannico, venuta a mancare a causa della «malafede crudele del tedesco» ${ }^{12}$. Per farlo si crearono cliché appositi, come il tradimento tedesco ad El Alamein o nelle pianure del Don. A questa ricostruzione ribatteva Mussolini da Radio Monaco, accusando la monarchia di tradimento. Quindi, chi ha tradito la patria? È questo il titolo del secondo capitolo, che ci introduce nel tema complesso della disputa tra fascismo e antifascismo. Da parte antifascista era Mussolini che aveva tradito la patria imponendo un'alleanza innaturale con la Germania di Hitler, da Salò invece era l'armistizio che veniva considerato tradimento della nazione e dell'alleato tedesco. Tuttavia, la narrazione assumeva forme di volta in volta diverse all'interno dello schieramento monarchico e dell'opposizione antifascista. La monarchia, infatti, doveva ristabilire la fiducia e la collaborazione con gli alleati; il fronte antifascista, invece, denunciava anche il «re fuggiasco» e «rivendicava per sé il diritto di guidare la "lotta di liberazione nazionale" contro l'invasore tedesco» ${ }^{13}$. Nonostante ciò vi erano alcuni temi fondamentali che non potevano essere diversamente elaborati: la guerra era di Mussolini e non degli italiani; Mussolini aveva tradito la patria con un'alleanza

\footnotetext{
9 FOCARDI, Filippo, Il cattivo tedesco e il bravo italiano. La rimozione delle colpe della seconda guerra mondiale, Roma-Bari, Laterza, 2013, p. IX.

${ }^{10}$ DEL BOCA, Angelo (a cura di), La storia negata. Il revisionismo e il suo uso politico, Vicenza, Neri Pozza, 2009, p. 9.

${ }^{11}$ LEONI, Giuliano, TAPPI, Andrea, «Pagine perse. Il colonialismo nei manuali di storia dal dopoguerra a oggi», in Zapruder, 23, 3/2010, pp. 154-167.

${ }_{12}$ FOCARDI, Filippo, Il cattivo tedesco e il bravo italiano, cit., p. 8.

13 Ibidem, p. 27.
} 
infausta. Temi su cui insisteva la propaganda alleata, che la monarchia cercò abilmente di sfruttare, ma che nell'antifascismo avevano ben diversa credibilità.

L'insistenza su queste argomentazioni era necessaria sia alla monarchia che alle forze antifasciste per cercare legittimazione nei confronti degli alleati, che lasciavano pochi margini di azione politica. Nel costruire il Tedesco come nemico storico, monarchici e antifascisti attinsero a piene mani dalla tradizione risorgimentale, cercando di far passare la Seconda guerra mondiale come l'ultima guerra di indipendenza, usando quella retorica anche per mobilitare il popolo contro l'invasore e contro Mussolini, che per anni aveva marchiato i partiti antifascisti di antipatriottismo. Ma tutto questo serviva soprattutto per riscattare l'Italia in vista dei trattati di pace, in modo da passare da cobelligeranti ad alleati paritari. Questo l'argomento sviluppato nel quarto capitolo, che ci mostra le diverse posizioni rispetto all'espiazione del popolo italiano. Da una parte le sinistre, per le quali la "vera Italia" era quella dei ceti operai che combattevano e avevano sempre combattuto il fascismo; dall'altra i moderati, che identificavano la "vera Italia" con una generica "Italia del Risorgimento". Nonostante queste differenze, il fattore comune era «la negazione dell'esistenza di un consenso popolare al regime e l'affermazione di una perfetta linea di continuità [...] fra la resistenza opposta al fascismo nel ventennio e la battaglia finale contro di esso ingaggiata dopo l'8 settembre»14. Da qui, quindi, l'identificazione del popolo italiano come vittima del fascismo, e da qui la distinzione tra Italia del Risorgimento e Germania di Bismarck. Considerazioni che portavano tanto gli antifascisti quanto la destra qualunquista alla richiesta di un «trattamento di riguardo da parte degli alleati»15. Facendo ciò, però, si oscurava volutamente il carattere più tragico di guerra civile e la realtà che il fascismo, lungi dall'essere un fenomeno minoritario, era sostenuto da un consenso di massa.

Se l'identificazione del popolo italiano come vittima del fascismo ebbe modo di trovare discordanze nelle diverse rappresentazioni, del tutto univoca fu invece la valutazione dell'alleanza con Hitler. Dimenticare l'asse, così il titolo del quinto capitolo, sintetizza la costruzione di un cliché comune, dai monarchici agli antifascisti ai qualunquisti, sulla definizione dell'alleanza come un fatto privato di Mussolini. Considerazione surrogata da Dino Grandi e ancor di più dai diari di Ciano e poi suffragata anche da uno storico come Barbagallo e persino da Palmiro Togliatti. In questo modo si mise in atto un processo che l'autore definisce «personalizzazione della

14 Ibidem, p. 53.

15 Ibidem, p. 64. 
colpa» ${ }^{16}$ in cui il popolo italiano viene nuovamente estraniato dal fenomeno fascista, il cui responsabile nelle sue più tragiche manifestazioni (leggi antiebraiche, alleanza con Hitler, guerre di occupazioni) viene ad essere identificato unicamente in Mussolini. In questo contesto - una guerra "non voluta né sentita" - il soldato «venne raffigurato come l'epitome della sofferenza»17. I soldati vennero ricordati indistintamente, vittime anch'essi della dittatura di Mussolini. Viceversa fu demonizzato il comportamento delle truppe tedesche, contribuendo a forgiare l'immagine del barbaro Tedesco, contrapposto al mite Italiano. L'impostazione del discorso ebbe però strutture diverse a seconda degli schieramenti. Se la monarchia usò questo topos per autolegittimarsi ed assolvere il proprio operato, le sinistre «intesero invece rimarcare la perniciosità della prevaricazione germanica quale aggravante delle colpe della corona e degli altri comandi militari» ${ }^{18}$, lasciando tuttavia invariata l'identificazione fittizia del "bravo italiano".

Nel sesto capitolo l'autore affronta più sistematicamente la distinzione tra "bravo italiano" e "cattivo tedesco". Così facendo, ci fa viaggiare tra gli scritti di Salvemini che si rallegra di notizie ricevute dalla Grecia sulla bontà dell'operato del soldato italiano, di Curzio Malaparte che tanto si spende nel descrivere i crimini tedeschi e di Benedetto Croce che sottolinea la differenza tra tedeschi, macchine inumane, e italiani che, viceversa, avevano mantenuto la propria umanità. D’altronde queste posizioni furono sostenute come strategia difensiva italiana ed è qui che riscontriamo un aspro scontro tra gli schieramenti in gioco. Se da una parte un quotidiano democristiano, «Il Popolo», pubblicava il 20 marzo 1946 (alla vigilia dell'apertura della conferenza del Consiglio dei Ministri degli Esteri delle quattro potenze) un articolo dal titolo I soldati difendevano $i$ greci contro la prepotenza tedesca con un occhiello che recitava Criminali di guerra noi?, in cui venivano elogiate le truppe italiane in Grecia, dall'altra «L'Italia Libera», giornale ufficiale del Partito d'Azione, sosteneva l'epurazione e denunciava i crimini commessi in Jugoslavia. Tuttavia le posizioni di quest'ultimo scatenarono la reazione del giornale monarchico «Italia Nuova» che accusava «L'Italia Libera» di trascurare gli «interessi materiali e morali» della Nazione ${ }^{19}$. Tuttavia, dopo la fine della guerra, l'atteggiamento delle sinistre mutò. Spinte dal contesto (la questione del confine orientale) ripiegarono nell'autodifesa nazionale, con un PCI oscillante tra posizioni divergenti. Da una parte, sulla stampa, i comunisti rivendicavano la necessità di un'estradizione dei criminali di guerra; dall'altra, presso le istituzioni, «condivisero

${ }^{16}$ Ibidem, p. 85 .

17 Ibidem, p. 90.

18 Ibidem, p. 99.

19 Ibidem, pp. 125-138. 
invece senza tentennamenti la posizione dei governi di unità antifascista contrari alla consegna dei presunti responsabili» ${ }^{20}$. Cosicché ancor oggi si sente il peso di una mancata "Norimberga italiana" e della difficoltà di parlare pubblicamente dei crimini di guerra italiani. C'è infatti in agguato una pericolosa inversione dei valori in riferimento alle diverse tragedie del confine orientale di cui - dopo che il dibattito è stato per anni lasciato in secondo piano - oggi si parla in un unico senso, quello dei crimini titini ${ }^{21}$.

L'ultimo capitolo, infine, è dedicato interamente alla costruzione, nell'immaginario collettivo, della "belva nazista". Il soldato viene cosi disumanizzato: «anche i gesti quotidiani appaiono innaturali»22. In questo caso furono le testimonianze di chi aveva vissuto l'esperienza dell'oppressione tedesca a esser sentite maggiormente. In particolare quella dello storico Carocci, che osservava come «la brutalità dei nostri guardiani era veramente l'espressione del loro popolo»23. Tuttavia ci fu chi cercò di far passare un'immagine non così univoca del tedesco. Era impossibile non parlare, ad esempio, del tedesco disertore. Nonostante ciò non si riusciva a crearne una raffigurazione realistica, declassandolo ad uomo non abbastanza coraggioso da combattere il suo dittatore.

Le conclusioni dell'autore approdano quindi alla realtà odierna che trova, ad esempio, una destra neofascista che avalla la contrapposizione tra cattivo tedesco e bravo italiano (e in questo senso sono auspicabili future ricerche che approfondiscano organicamente la memoria perpetuata durante la fase repubblicana), nell'amara considerazione che in Italia è del tutto assente un'etica della responsabilità capace di uscire da una memoria «vittimistica e autocelebrativa». Il lavoro di Filippo Focardi è in questo senso un tassello significativo e necessario, portato a termine con una chiarezza espositiva e di sintesi (che non pregiudica la completezza del lavoro) a volte rara ${ }^{24}$, per comprendere le radici di una memoria che sarebbe ora che lasciasse spazio a riflessioni più responsabili e meno identitarie della storia.

\footnotetext{
20 Ibidem, p. 139.

${ }^{21}$ In merito al problema delle Foibe, diversi storici e studiosi sono tacciati di "revisionismo" o di "negazionismo", in particolare Alessandra Kersevan, Joze Pirjevec e Claudia Cernigoi. Per quanto le loro tesi possano essere discutibili, risulta difficile alla prova dei fatti usare lo stesso termine che identifica invece quel fenomeno che Vidal-Naquet ha definito di "assassinio della memoria".

${ }^{22}$ FOCARDI, Filippo, Il cattivo tedesco e il bravo italiano, cit., p.155.

23 Ibidem, pp. 162-163.

24 Nel giudizio caustico di Silvio Lanaro «gli storici scrivono mediamente male». Cfr. LANARO, Silvio, Raccontare la storia, Marsilio, Venezia, 2004, p. 143.
} 


\section{* L'autore}

Oreste Veronesi ha conseguito la laurea in Storia presso l'Università di Padova nel luglio 2013 con una tesi dal titolo La strage di Piazza Fontana nelle pagine de "L'Arena» (1969-1972). Attualmente è iscritto al corso di inter-ateneo Verona - Trento in Scienze storiche.

URL: $<$ http://www.studistorici.com/progett/autori/\#Veronesi >

\section{Per citare questo articolo:}

VERONESI, Oreste, «Recensione: Filippo FOCARDI, II cattivo tedesco e il bravo italiano. La rimozione delle colpe della seconda guerra mondiale, Roma - Bari, Laterza, 2013, XIX + 288 pp.», Diacronie. Studi di Storia Contemporanea : Spazi, percorsi e memorie, 29/10/2013,

URL:< http://www.studistorici.com/2013/10/29/veronesi_numero_15/ >

Diacronie Studi di Storia Contemporanea of www.diacronie.it

Risorsa digitale indipendente a carattere storiografico. Uscita trimestrale. redazione.diacronie@hotmail.it

Comitato di redazione: Marco Abram - Jacopo Bassi - Luca Bufarale - Alessandro Cattunar - Elisa Grandi - Deborah Paci - Fausto Pietrancosta - Matteo Tomasoni - Luca Zuccolo

Diritti: gli articoli di Diacronie. Studi di Storia Contemporanea sono pubblicati sotto licenza Creative Commons 2.5. Possono essere riprodotti a patto di non modificarne i contenuti e di non usarli per fini commerciali. La citazione di estratti è comunque sempre autorizzata, nei limiti previsti dalla legge. 